\title{
Fixer l'équivalence terminologique sur l'exemple des termes français et polonais du régime supplémentaire de retraite
}

\author{
To establish terminological equivalence on the basis of French and Polish \\ terms in individual retirement plans
}

\author{
Liliana Kozar \\ Uniwersytet Zielonogórski \\ lkozar@poczta.onet.pl
}

\begin{abstract}
This paper constitutes a kind of protocol, based on the author's experience, which proposes to review several situations that occurred during her attempt to determine equivalence between French and Polish terms in the field of individual retirement plans. This attempt was made exclusively on various textual sources and their related data allowed to isolate a number of situations that differ in their details.
\end{abstract}

Keywords: term, terminological equivalence, definition, semasiological approach, onomasiological approach

La mondialisation des savoirs et des savoir-faire spécialisés force une élaboration continue des outils terminologiques bilingues et plurilingues sous forme des dictionnaires, glossaires ou bases de données. De plus en plus nombreux qu'ils sont, ils présentent sans réserve des disparités : soit leur disponibilité est réduite à quelques domaines dominants, soit, s'ils existent, ils ne couvrent pas tout le champ de référence, soit encore ils ne satisfont pas à tous les besoins de la traduction. Tôt ou tard, tout traducteur consciencieux dans son activité est amené à entreprendre une recherche terminologique ayant entre autres pour but d'établir la relation d'équivalence entre les termes appartenant à des langues visées. Une tâche loin d'être facile, étant donné que le parallélisme entre les descripteurs textuels, que fournissent les sources, n'est pas toujours garanti.

Le présent article constitue une sorte de protocole se proposant, sur les traces de notre expérience personnelle, de passer brièvement en revue plusieurs 
situations identifiées lors de l'appariement des termes français et polonais appartenant au domaine du régime supplémentaire de retraite ; il constitue donc un relevé de cas de figure qui divergent dans leurs détails.

1. Le corpus des termes servant à notre démonstration ${ }^{1}$ a été confectionné, tout comme un certain nombre d'informations relatives à ces termes, à partir d'un dépouillement des ressources textuelles ${ }^{2}$, et répertorié sous forme de fiches terminologiques, initialement unilingues. Une telle disposition a permis, à l'encontre des listes des termes, d'avoisiner dans des rubriques distinctes un certain nombre de données d'ordre encyclopédique (définition, contexte définitoire, note, etc.) et/ou linguistique (collocation, synonyme, antonyme, variante, etc.), concernant un terme et, en conséquence, de le voir comme une entité terminologique ayant une existence hors et dans la langue. La structure des fiches est variable et correspond à autant de catégories de données apportant pour chaque terme un type d'information spécifique (cf. Gouadec, 1990:38), soulignons-le, cernée dans les textes. En effet, cette confection, menée séparément pour chaque langue visée, a révélé de nombreuses contiguïtés entre les deux systèmes notionnels étudiés - des systèmes grosso modo semblables, toutefois non identiques $^{3}$ - en éveillant une forte sollicitude qui alors se montrait toute naturelle : tenter, dans la mesure du possible, de fixer l'équivalence sur l'ensemble terminologique retenu, d'autant plus qu'à notre connaissance la terminologie du domaine n'a jamais été soumise à une telle tentative.

2. La relation d'équivalence entre deux termes est notamment fondée sur le parallélisme dans la relation terme $\Leftrightarrow$ notion, établie séparément pour chacune des langues visées. La démarche initiale paraît donc sémasiologique et consiste à aller de la dénomination identifiée vers le concept qu'elle désigne ; celui-ci, au mieux, saisi dans une définition qui devrait préserver un ensemble de caractères distinctifs et pertinents, inhérents au concept et permettant de le distinguer avec précision parmi d'autres. De là, une approche d'emblée onomasiologique intervient qui consiste à confronter les définitions en deux langues ; la convergence au niveau des caractères permet « de conclure à l'équivalence totale ou partielle des termes » (de Bessé, 1990 : 260).

\footnotetext{
${ }^{1}$ Au bilan, près de 800 termes français et plus de 750 termes polonais, simples et composés, sans compter les variantes et synonymes.

${ }^{2}$ Il s'agit de textes de différents nature, longueur et degré de spécialisation, ayant pourtant un cadrage temporel d'une dizaine d'années.

${ }^{3}$ Précisons que le régime supplémentaire de retraie, appelé tout court le troisième pilier de la retraite, en France et en Pologne, fait partie d'une construction plus complexe qu'est le système de retraite. Il s'agit en effet d'un vaste domaine qui peut en pratique référer à toute initiative financière individuelle visant à se constituer un revenu complémentaire, suppléant un jour la pension de retraite.
} 


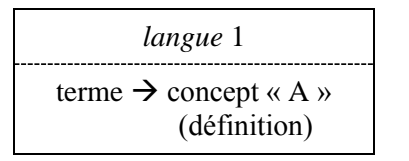

\begin{tabular}{|c|c|c|}
\hline ᄃ & \multicolumn{2}{|c|}{ langue 2} \\
\hline & $\begin{array}{c}\text { concept «A } \text { (définition) }\end{array}$ & $\begin{array}{l}\rightarrow \quad \text { terme } \\
\text { (terme équivalent) }\end{array}$ \\
\hline
\end{tabular}

La qualité d'une telle activité terminologique dépend largement de la qualité des définitions répertoriées dans les sources. L'idéal, quand elles facilitent la transmission des connaissances sur le concept, en délimitant son champ notionnel $^{4}$; quand elles insèrent ce concept à l'intérieur d'un champ notionnel et permettent de reconstruire, dans une certaine mesure, l'organisation de ce champ ; et l'essentiel, quand, en confrontant les définitions en deux langues, on a affaire à des définitions harmonisées, dans le sens que leur attribue Loïc Depecker (2000 : 116-117) :

La définition harmonisée (...) est une définition de concept (...) susceptible de permettre sa représentation linguistique dans les langues traitées ou visées. Elle doit former le pivot autour duquel ces langues peuvent s'ajuster.

Il s'avère toutefois qu'à côté des définitions qui paraissent satisfaisantes du point de vue terminologique et qui permettent sans la moindre gêne d'établir la relation d'équivalence, les textes fournissent celles qui présentent de nombreuses déficiences quant à la reconnaissance des caractères dits pertinents, déjà au niveau d'une seule langue ${ }^{5}$; des définitions visiblement insuffisantes ou partielles, voire défectives dont la valeur référentielle ou rédactionnelle peut être aisément mise en cause et dont la qualité peut en conséquence altérer les croisements des données au niveau des deux langues confrontées.

Par ailleurs, la définition n'est pas la seule donnée pouvant contribuer à l'appariement des termes. Toutes sortes d'informations qui «(...) correspondent à des contextes terminologiques ou segments de textes ou discours contenant des éléments de définition potentielle » (Gouadec, 1993: 74), donc des contextes définitoires, des contextes explicatifs, commentaires, contextes associatifs (Kaufman, 2004: 51) sont des indices qui contribuent à la construction progressive de la représentation plus ou moins complète du concept et, si le but est tel, à la détermination de l'équivalence. Pourtant, partout où ces

\footnotetext{
${ }^{4}$ Pourquoi pas par rapport à d'autres concepts.

${ }^{5}$ Il convient de souligner que la spécificité du domaine retenu n'est pas à cet égard notre bon allié, car il s'agit en effet d'une réalité largement abstraite, reconnaissable à travers les mécanismes économiques fort complexes, les opérations actuarielles d'une grande technicité ; d'une réalité qui élabore plutôt des «idéalités » sans rapport direct avec une représentation mentale quelconque.
} 
données sont lacunaires ou disparates, fixer cette relation de manière irrévocable est une opération fort risquée.

3. Pour en parler plus en détails, en voici un relevé, non exhaustif, de situations rencontrées lors de la l'appariement des termes français et polonais appartenant au domaine de référence ${ }^{6}$ :

3.1. Parmi les termes mis en parallèle, il y en a ceux qui répondent aux exigences de l'équivalence totale, car leur référenciation en deux langues est isomorphe. Cet isomorphisme est confirmé soit (a) par la définition (il s'agit donc largement de définitions harmonisées), soit (b) par d'autres données équivalentes contenues sur la fiche :

(a) prime annuelle

« Prime versée une fois par an »

année d'assurance

«Période de 12 mois qui sépare deux anniversaires consécutifs de la date d'effet »

(b) Co-souscription

«Deux personnes peuvent souscrire en commun un contrat d'assurance vie. Chaque co-souscripteur se nomme bénéficiaire en cas de vie et désigne l'autre cosouscripteur bénéficiaire en cas de décès. Le dénouement est prévu au premier ou au deuxième décès »

prime unique

«Un seul versement initial, effectué à la souscription $»$ sktadka roczna

„Składka regulowana raz w roku”

rok polisowy

„Okres upływający pomiędzy kolejnymi rocznicami polisy"

ubezpieczenie na dwa życia

„Ubezpieczenie obejmujące ochroną ubezpieczeniową dwie osoby. Wyróżnić można dwa typy takich produktów: 1) gdy świadczenie $\mathrm{z}$ tytułu ubezpieczenia wypłacane jest w przypadku pierwszego zgonu jednej z ubezpieczonych osób, 2) gdy świadczenie wypłacane jest dopiero $\mathrm{z}$ tytułu zgonu ostatniej z ubezpieczonych osób"

sktadka jednorazowa

„Ubezpieczający ma prawo zawrzeć umowę ze składką jednorazową opłacaną z góry za cały okres ubezpieczenia"

3.2. Il arrive souvent que les informations retenues, bien qu'elles décrivent le même concept, ne se recouvrent pas en deux langues à cent pour cent ou s'écartent en pointant sur des traits différents. De telles situations, d'ailleurs non isolées, induisent que soit chacune des langues conceptualise les mêmes notions différemment, soit la différence réside dans la manière d'appréhender et de

\footnotetext{
${ }^{6}$ Les données figurant dans ce relevé sont telles qu'elles étaient extraites des ressources.
} 
décrire le concept par l'auteur de l'ouvrage où l'information a été puisée ${ }^{7}$, soit encore les données recueillies sont toujours incomplètes et par conséquent incompatibles. Sans pouvoir exclure chacune de ces possibilités et d'en identifier clairement la nature, nous trouvons légitime de considérer un certain groupe de termes appariés à base d'informations tantôt non-homologues, tantôt insuffisantes ou fragmentaires, comme équivalents totaux, parce qu'ils possèdent comme dénominateur commun, nous en sommes persuadée, le même concept ; leur référenciation est donc identique.

Par ailleurs, Daniel Gouadec (1993: 128) qualifie ce type de relation de référenciation fonctionnelle qui «s'entend au sens où la 'pertinence' technique est la même bien que les informations fournies par les définitions puissent différer ». Cela induit que si une même notion est identifiable dans les deux systèmes notionnels, chacun propre à une langue, son sens est le même, quoique possiblement conceptualisé différemment. La saisie du sens peut donc dépasser le cadre rédactionnel d'une définition et se préciser dans les conditions contextuelles ou discursives, explicites ou implicites. Toutefois, dans cette matière délicate, il faut être particulièrement vigilant.

De la sorte, tendons à considérer les unités citées ci-dessous en termes d'équivalence totale, malgré les disparités plus ou moins apparentes dans la rédaction de leurs descripteurs, que ce soit la définition ou autre :

action au porteur

« Titre de propriété où le nom du détenteur n'apparaît pas »

participation aux bénéfices

« Rémunération complémentaire ajoutée audelà de garantie par certaines compagnies d'assurance » akcja na okaziciela

„Akcja nie zawierająca danych osobowych akcjonariusza, której zbywalność nie może zostać ograniczona, a sprzedaż nie wymaga zachowania szczególnej formy"

udziat $w$ zysku

„Oprócz sumy ubezpieczenia określonej w umowie, wypłacany jest udział w zysku $\mathrm{z}$ tytułu dochodów $\mathrm{z}$ inwestycji funduszy ubezpieczeniowych"

3.3. Lors du travail entrepris, nous étions souvent confrontée au manque de coïncidence parfaite entre les significations des termes en deux langues, dû

${ }^{7}$ Par une telle formulation, nous remettons en question, d'ailleurs après plusieurs auteurs (cf. Gouadec, 1990 ; Béjoint, Thoiron, 2000 ; Kaufman, 2002), l'un des principes fondamentaux de la terminologie classique, celui de l'objectivité des données terminologiques et de la neutralité du discours terminologique. 
notamment aux différences non négligeables entre les deux systèmes notionnels mis en relation ${ }^{8}$. Par ailleurs, les études cognitives démontrent que la conceptualisation des faits spécialisés ne semble pas être la même selon les communautés linguistiques (Béjoint, Thoiron, 2000 : 14-15), et que la correspondance entre les notions d'une langue à celles d'une autre n'égale que rarement $1: 1$ (Kozłowska, 2007 : 56). Pour cause, il ne s’agit pas de deux systèmes idéalement superposables, mais se correspondant. Dans toutes ces situations, il est équitable de parler de l'équivalence partielle, bien que le terme, lui-même, incorpore plusieurs cas spécifiques.

3.3.1. Parfois, malgré de fortes prémisses informant sur la relation d'équivalence entre deux termes, notamment dictées par l'identification du concept, il peut s'agir simplement d'une différence dans sa compréhension ou sa conceptualisation, ce qui étonne en effet Marc Van Campenhoudt (2000 : 137-138):

(...) La notion apparaît autant comme un fait de langue et de culture que comme le résultat d'une pure opération mentale. Dans la logique d'une perspective strictement conceptuelle, la notion correspond à un ensemble de caractères qui constituent des propriétés de l'objet conceptualisé et sont censément compatibles d'une langue à l'autre. Peut-on encore soutenir que les caractères émanent de l'objet et ne sont pas conditionnés par la langue envisagée?

Il semble que la réponse à la question ainsi posée soit doctrinairement incorrecte. Car il s'avère effectivement, les exemples surabondent, que la conceptualisation, notamment des concepts abstraits, peut être ou est souvent une affaire de langue :

prime nette

« Prime ne comprenant pas de taxe sur les opérations d'assurance » sktadka netto

„Część składki, która zasila fundusz przeznaczony na wypłatę przez zakład ubezpieczeń pieniężnych świadczeń ubezpieczeniowych"

\footnotetext{
${ }^{8}$ Le problème d'équivalence face aux divergences notionnelles entre les langues a depuis des années intéressé les terminologues. Il paraît qu'à cet égard la vision d'Eugène Wüster, reprise et cultivée par tant d'éminents chercheurs (Rey 1992: 47s., Kozłowska 2007: 118s., Lukszyn, Zmarzer $2006: 85 \mathrm{~s}$.), tendant vers un idéal monosémique et monoréférentiel international et résultant d'un interventionnisme normatif dans les systèmes notionnels afin de les rendre dans la mesure du possible isomorphes, reste répandue. Les recommandations d'ISO ou d'autres organismes ayant la faculté d'influer sur la matière terminologique afin de garantir son développement standardisé, tels que les commissions ministérielles de terminologie ou comités de normalisation mis en place, en sont la preuve.
} 
avis de situation

« Document précisant les valeurs de rachat et de réduction d'un contrat d'assurance vie, que l'assureur doit adresser chaque année au souscripteur » pakiet rocznicowy

„Coroczna informacja o wartości polisy”

3.3.2. Parfois, il peut s'agir de différences tantôt ponctuelles, tantôt techniques, inhérents à chaque système ou à la culture de référence :

retraite réversible

« En cas de décès de l'affilié, 60\% des droits acquis sont versés à son conjoint ou à toute autre personne librement désignée »

pension de base

« Rémunération calculée sur la moyenne du revenu pendant les quatorze meilleures années de la carrière versée par le Régime de base ») emerytura matżeńska

„Mężowi i żonie wypłaca się świadczenie W określonej wysokości A aż do śmierci pierwszego $\mathrm{z}$ nich, a potem $\mathrm{w}$ wysokości B owdowiałej osobie, aż do jej śmierci”

emerytura podstawowa

„Świadczenie społeczne wypłacane z ZUS-u po osiągnięciu wieku emerytalnego"

3.3.3. Il se peut que les mots définisseurs employés dans les définitions appartiennent visiblement à des classes sémantiques différentes et, par là, recouvrent possiblement une aire conceptuelle différente ou communiquent des concepts distincts. Il peut s'agir alors du même phénomène mais envisagé en quelques sortes d'un autre point de vue ou de la polysémie générée par la métonymie classant le terme dans une catégorie notionnelle différente. À défaut d'informations suffisantes pour pouvoir exclure l'une ou l'autre possibilité, les termes concernés se trouvent provisoirement juxtaposés :

prise d'effet du contrat

«Date à laquelle un contrat d'assurance entre en vigueur »

\section{retraite supplémentaire}

«Possibilité de verser les cotisations facultatives de manière à percevoir, au moment de la retraite une rente qui s'ajoutera aux pensions de base et complémentaires »

\section{materialny początek ubezpieczenia}

„Rozpoczęcie przez zakład ubezpieczeń świadczenia ochrony ubezpieczeniowej”

indywidualne zabezpieczenie emerytalne

„Uzupełnienie świadczeń emerytalnych otrzymywanych w ramach dwóch pierwszych filarów" 
3.3.4. Il arrive que certaines unités se distinguent de leurs homologues proposés par un sémantisme plus vaste, ce qui veut dire que l'aire conceptuelle d'un terme dans une langue peut recouvrir celles des deux termes, voire plus, dans l'autre et désigner effectivement un concept générique ${ }^{9}$; ou inversement, avoir une dimension sémantique plus limitée par rapport au terme suggéré équivalent et désigner un concept spécifique par rapport à celui-ci. Cette dissymétrie dans le fonctionnement des deux systèmes ou, plus précisément, le manque de concordance dans la distribution des caractères conduit à constater qu'alors on est toujours confronté à l'équivalence partielle. Les termes cidessous illustrent ce phénomène :

$\begin{array}{ll}\begin{array}{l}\text { intermédiation d'assurance } \\ \text { courtage d'assurance }\end{array} & \rightarrow \quad \text { pośrednictwo ubezpieczeniowe } \\ \text { affilié } \quad \text { (régime de base) } & \text { (régime de base) } \\ \text { assuré social } & \text { (régime complémentaire) } \\ \text { adhérent } & \text { (régime de base / régime } \\ \text { participant } & \text { complémentaire) } \\ \text { (assurance vie / régime } & \text { supplémentaire) } \\ \text { tête } & \begin{array}{l}\text { (régime de base / régime } \\ \text { complémentaire / régime } \\ \text { supplémentaire) }\end{array} \\ \text { assuré }\end{array}$

3.4. Il arrive que la relation d'équivalence établie pour certaines paires de termes puisse paraître fortuite ou potentielle. Malgré de fortes prémisses qu'il soit question d'une contiguïté entre les concepts désignés, l'équivalent suggéré peut ne pas satisfaire pleinement à la dimension conceptuelle du terme auquel il est joint. Il peut être par exemple de valeur générique ou spécifique, voire être par rapport au terme indiqué homologue en légère discordance. Bref, il peut s'agir des concepts proches, quoique non identiques. Dans toutes ces situations, l'équivalence, à défaut de parallélismes rigoureux qui peuvent la fixer sans ambiguïté, est statutairement incertaine ou temporairement provisoire, et ne signifie pas un choix final décisif. Force est d'entamer une recherche supplémentaire ou de solliciter l'intervention d'un spécialiste pour vérifier si,

\footnotetext{
${ }^{9}$ Marc Van Campenhoudt (1995: 6) emploie à cette occasion le terme d'hyperonomase.
} 
effectivement, il s'agit d'un manque de précision, des concepts différents ou d'une divergence réelle entre les langues. Ceci est entre autres le cas des termes :

questionnaire de risque

« Document que doit remplir l'assuré lors de la souscription d'un contrat »

note de couverture

« Document délivré par l'assureur en attendant l'établissement du contrat définitif » underwriting

„Postępowanie poprzedzające zawarcie umowy ubezpieczenia mające na celu oszacowanie wielkości przyjmowanego przez ubezpieczyciela ryzyka"

tymczasowa ochrona ubezpieczeniowa

„Tymczasowa ochrona rozpoczyna się w dniu doręczenia poprawnie wypełnionego wnios$\mathrm{ku}$ ubezpieczeniowego $\mathrm{z}$ dowodem wpłaty pierwszej składki do siedziby towarzystwa"

3.5. Certains termes figurent comme paires terminologiques probablement équivalentes. Il est question des unités qui n'ont pas été équipées d'un nombre d'informations satisfaisant en une langue. Toutefois, sur la base de quelques indices, l'opération d'appariement a été tentée. Parmi ces indices, il faut notamment citer la forme composée du terme grâce à laquelle celui-ci peut, certes dans une dimension limitée, s'autodéfinir ou s'expliciter :

antécédents

«Informations relatives au passé de l'assuré permettant à l'assureur de lui proposer une tarification, en fonction de l'évaluation des risques identifiés »

assiette de cotisation

«Base de calcul sur laquelle sont déterminées les cotisations »

médecin de la société d'assurance

prime semestrielle przeszłość ubezpieczeniowa

podstawa wymiaru składki

lekarz konsultant

„Lekarz autoryzowany przez ubezpieczyciela"

sktadka pótroczna

„Składka opłacana dwa razy do roku” 
3.6. Il arrive qu'une unité de dénomination, ou plus précisément ses descripteurs communiquent l'existence de plus d'un seul concept. L'hypothèse de travail est alors d'envisager l'existence potentielle de concepts distincts, possiblement reliés entre eux (habituellement par une relation de contiguïté), dénommés par une seule forme terminologique. La polysémie, dont il est question, pose inévitablement divers problèmes, d'abord au niveau de son identification et de son traitement, ensuite, au niveau de la mise en équivalence, car - il faut le rappeler - les langues n'approchent pas la réalité de la même manière. Marc Van Campenhoudt (1995: 2, 2000 : 133) apporte dans cette matière une proposition à envisager ${ }^{10}$. Son principe, dénommé le principe d'équivalence notionnelle (P.E.N.), peut se résumer dans le propos suivant :

Si A de $\mathrm{L}_{1}$ (langue 1) est équivalent à $\alpha$ de $\mathrm{L}_{2}$ (langue 2) et si $\mathrm{A}$ de la $\mathrm{L}_{1}$ est équivalent à $\beta$ de $L_{2}$ alors que $\alpha$ de $L_{2}$ n'est pas synonyme de $\beta$ de $L_{2}$, c'est que probablement $A$ de $\mathrm{L}_{1}$ possède deux sens (...).

\begin{tabular}{|l|l|l|}
\cline { 2 - 3 } \multicolumn{1}{c|}{} & L1 & L2 \\
\hline Entrée 1 & A & $\alpha$ \\
\hline Entrée 2 & A & $\beta$ \\
\hline
\end{tabular}

L'approche ainsi définie visant à identifier et à apparier les termes, ici polysémiques, se montre en même temps logique et naturelle. À partir de l'observation des dénominations de chacune des langues, de l'identification des concepts qu'elles véhiculent (sémasiologie), on passe à la recherche des équivalents. Cette recherche doit respecter autant que possible les faits décrits (onomasiologie) (Van Campenhoudt, 1995: 2), car l'équivalence ne s'établit qu'au niveau des concepts. Quoique la dialectique d'une telle conduite paraisse nette, la réalité terminologique se montre beaucoup plus complexe. Pour illustrer quelques relations qui s'établissent entre les termes identifiés dans notre corpus comme polysémiques, les concepts qu'ils désignent et ensuite les équivalents que ces concepts véhiculent, nous avons adopté un système de symboles qui permettra, nous l'espérons, de saisir plus efficacement les nuances qui s'imposent $^{11}$ :

${ }^{10}$ Sa proposition est en opposition à l'approche classique normalisatrice. De notre position d'un observateur qui essaie d'enregistrer les faits terminologiques de manière objective, tels qu'ils se présentent dans les textes, elle paraît d'emblée intéressante.

${ }^{11}$ De manière plus explicite, dans ce système, le terme français sera représenté par une lettre de l'alphabet latin, tandis que son équivalent polonais proposé par une lettre grecque ; les chiffres : 
3.6.1. Il arrive que les concepts qui partagent une même dénomination en une langue équivalent aux concepts qui, eux aussi, sont dénommés par un terme polysémique en seconde langue. Le caractère linéaire de la polysémie en deux langues, quoique rare, est donc possible et la relation qui s'établit alors pourrait être représentée de manière suivante:

$$
\mathrm{A}_{(1)} \Leftrightarrow \alpha_{(1) ;} \quad \mathrm{A}_{(2)} \Leftrightarrow \alpha_{(2) ;} \quad \mathrm{A}_{(3)} \Leftrightarrow \alpha_{(3)}
$$

assurance (1)

«Opération par laquelle l'assureur, en contrepartie d'une cotisation, promet à l'assuré ou à un tiers une garantie en cas de réalisation du risque assuré »

assurance (2)

« Dispositif d'indemnisation des dommages aux biens et aux personnes par la prise en charge des risques et de leur compensation »

assurance (3)

«Contrat d'assurance par lequel, en contrepartie du versement d'une prime le souscripteur se fait promettre pour son compte ou celui d'un tiers, par l'assureur une prestation pécuniaire en cas de réalisation d'un risque » ubezpieczenie (1)

„Działanie, które z jednej strony na podstawie umowy i dzięki opłacie składki pozwala zakładowi ubezpieczeń przyjąć na siebie, a ubezpieczającemu przekazać do zakładu ryzyko negatywnych, ekonomicznych skutków określonego zdarzenia losowego"

ubezpieczenie (2)

„Instrument pozwalający zastąpić wielką, ale niepewną stratę powstałą w wyniku zdarzenia losowego, stosunkowo niewielką, ale pewną stratą, składką ubezpieczeniową"

ubezpieczenie (3)

„Umowa ubezpieczenia, na podstawie której zakład ubezpieczeń zobowiązuje się spełnić określone świadczenie $\mathrm{w}$ razie zajścia przewidzianego $\mathrm{w}$ umowie wypadku, a ubezpieczający zobowiązuje się zapłacić składkę"

3.6.2. Il arrive que la terminologie polonaise, faute de dénominations indigènes, emprunte à un système terminologique primaire, anglais ou, comme le montre l'exemple cité, français, un terme polysémique pour dénommer des concepts analogues, synchroniquement nouveaux. La relation d'équivalence qui s'établit alors entre les termes reste isomorphe:

$$
\mathrm{A}_{(1)} \Leftrightarrow \alpha_{(1)} ; \quad \mathrm{A}_{(2)} \Leftrightarrow \alpha_{(2)}
$$

bancassurance (1)

«Terme désignant les synergies qui peuvent se créer entre la banque et l'assurance:

\section{bancassurance (1)}

„Aktywne uczestnictwo banków w sprzedaży polis ubezpieczenia na życie, które polega na

(1), (2), (3), (4) renseigneront sur le nombre des concepts identifiés et véhiculés par une même dénomination. 
rapprochement entre une banque et une société d'assurance, afin d'accroître leurs fonds propres et leurs capacités d'intervention sur les marchés financiers et dans l'économie »

bancassurance (2)

«Vente des produits d'assurance par des banquiers » połączeniu usług oraz sprzedaży wspólnych produktów przez bank i towarzystwo ubezpieczeniowe"

bancassurance (2)

„Sprzedaż produktów ubezpieczeniowych przez banki”

3.6.3. Il arrive qu'un terme polysémique français désigne des concepts distincts contigus auxquels peuvent correspondre des concepts distincts contigus désignés par une unité polonaise. Une telle formulation n'apporterait rien si on admettait une relation linéaire entre les concepts mis en relation en deux langues; toutefois, il arrive que malgré certaines similarités, la relation d'équivalence soit perturbée, comme ci-dessous :

actuariat (1)

«Service en charge des travaux actuariels (réalisés par un actuaire) »

actuariat (2)

«Fonction de l'actuaire » aktuariat (1)

„Ogół zastosowań matematyki w ubezpieczeniach"

aktuariat (2)

„Działalność aktuariusza”

Si au niveau des termes actuariat (2) $\Leftrightarrow$ aktuariat (2) l'équivalence ne pose pas de doute, au niveau de actuariat $(1) \approx$ aktuariat (1), elle ne se laisse pas unanimement démontrer. Les définitions jointes s'opposent visiblement. Cela peut induire que soit (a) les termes se correspondent, toutefois leur conceptualisation en deux langues se déploie différemment, soit (b) il faudrait peut-être considérer l'existence d'un concept qui fonctionne en français et non en polonais, et vice versa, l'existence d'un concept dans le système polonais, méconnu dans le système français, soit encore (c) il faudrait envisager pour chacun des systèmes l'existence des trois concepts symétriques qui pour le moment n'ont pas été identifiés.
(a) $\mathrm{A}_{(1)} \Leftrightarrow \alpha_{(1)}$
(b) $\mathrm{A}_{(1)} \Leftrightarrow \varnothing_{\text {(vide notionnel) }}$
(c) $\mathrm{A}_{(1)} \Leftrightarrow \alpha_{(1)}$
$\mathrm{A}_{(2)} \Leftrightarrow \alpha_{(2)}$
$\varnothing \Leftrightarrow \alpha_{(1)}$
$\mathrm{A}_{(2)} \Leftrightarrow \alpha_{(2)}$
$\mathrm{A}_{(2)} \Leftrightarrow \alpha_{(2)}$
$\mathrm{A}_{(3)} \Leftrightarrow \alpha_{(3)}$ 
$\mathrm{Vu}$ que les recherches supplémentaires entreprises n'ont pas apporté de réponses à ces questions et sans pouvoir exclure chacune des possibilités, le statut d'équivalence pour actuariat (1) et aktuariat (1) reste pour le moment incertain.

3.6.4. Il arrive qu'un terme polysémique français désignant des concepts différents reçoive comme équivalents les termes polonais distincts dans leurs formes :$$
\mathrm{A}_{(1)} \Leftrightarrow \alpha
$$ \\ actifs (1) \\ «Personnes en activités » \\ actifs (2) \\ "Choix des différents actifs composant le \\ patrimoine »
}

allocation (1)

« Prestation à caractère sociale »

allocation (2)

«C'est au propriétaire du contrat de choisir librement les titres »
$\mathrm{A}_{(2)} \Leftrightarrow \beta$

osoby czynne zawodowo

„Osoby aktywne zawodowo"

aktywa

„Ogół środków finansowych i niefinansowych, którymi dysponuje w danym momencie zakład"

zasitek

„Świadczenie pieniężne wypłacane jako forma pomocy osobom, które czasowo lub trwale nie są w stanie zaspokoić swoich materialnych potrzeb"

alokacja sktadki

„Określony w polisie udział procentowy w składce przeznaczony na zakup jednostek funduszy"

3.6.5. Il arrive qu'à un terme polysémique français désignant plusieurs concepts autonomes contigus correspondent deux termes distincts dans leurs formes, à leur tour polysémiques. Cette distinction peut par exemple résider dans l'opposition singulier/pluriel :

$$
\mathrm{A}_{(1)} \Leftrightarrow \alpha_{(1) ;} \quad \mathrm{A}_{(2)} \Leftrightarrow \alpha_{(2)} ; \quad \mathrm{A}_{(3)} \Leftrightarrow \beta_{(1) ;} \quad \mathrm{A}_{(4)} \Leftrightarrow \beta_{(2) ;}
$$

assurance vie (1)

ubezpieczenia na życie (1)

«Branche des assurances individuelles ou souscrites dans le cadre des assurances de groupe »

„Dział ubezpieczeń, do którego zalicza się: ubezpieczenie na życie, ubezpieczenie posagowe, zaopatrzenia dzieci, ubezpieczenie na życie związane z funduszem inwestycyjnym, ubezpieczenie rentowe, ubezpieczenie wypadkowe i chorobowe, jeżeli są uzupełnieniem wymienionych ubezpieczeń" 
assurance vie (2)

«Assurances qui réunit deux catégories de produits : l'assurance vie (placement, épargne, retraite, ...) qui garantissent à l'échéance du contrat, le versement à l'assuré d'un capital augmenté des intérêts, et l'assurance décès qui garanti le versement d'un capital à un bénéficiaire désigné en cas de décès de l'assuré »

assurance vie (3)

« Contrat souscrit en vu de se constituer un supplément de retraite sous forme d'un capital ou d'une rente »

assurance vie (4)

«Placement ou produit de long terme géré par un organisme financier, récupérable sous forme de capital ou de rente » ubezpieczenia na życie (2)

„Ubezpieczenia, przedmiotem których są niemajątkowe, osobiste dobra człowieka, tj. jego życie i zdrowie"

ubezpieczenie na życie (1)

„Umowa, przez którą zakład ubezpieczeń zobowiązuje się do spełnienia świadczenia w postaci zapłaty umówionej sumy pieniężnej, renty albo innego świadczenia, w razie zajścia przewidzianego $\mathrm{w}$ umowie wypadku w życiu osoby ubezpieczonej”

ubezpieczenie na życie (2)

„Ubezpieczenie na życie to inwestycja długoterminowa; korzystna forma oszczędzania na emeryturę"

3.6.6. Il arrive qu'un terme polysémique français désignant des concepts autonomes reçoive comme équivalents deux termes polonais formellement distincts dont l'un possède une plus grande extension et est par rapport à lui en relation générique ou, si nous recourions au terme employé par Marc Van Campenhoudt (1995 : 6s.), en relation d'hyperonomase.

$$
\mathrm{A}_{(1)} \leq \alpha_{(1) ;} \quad \mathrm{A}_{(2)} \Leftrightarrow \beta ;
$$

assurance vieillesse (1)

ubezpieczenie emerytalne

«Branche de la protection sociale vieillesse française qui oblige chacun à cotiser en vue de percevoir une pension à vie au moment de sa retraite »

assurance vieillesse (2)

skladka na fundusz emerytalny

«Contribution sociale sur le revenu qui oblige chacun à cotiser en vue de percevoir une pension à vie au moment de la retraite » 
3.6.7. Il arrive que la relation d'équivalence qui s'établit entre les termes polysémiques des deux langues soit plus complexe :

$$
\mathrm{A} \Leftrightarrow \alpha_{(1) ;} \quad \mathrm{B}_{(1)} \Leftrightarrow \alpha_{(2) ;} \quad \mathrm{B}_{(2)} \Leftrightarrow \alpha_{(3) ;} \quad \mathrm{B}_{(3)} \Leftrightarrow \alpha_{(4) ;}
$$

assurance vieillesse

«Branche de la protection sociale vieillesse française qui oblige chacun à cotiser en vue de percevoir une pension à vie au moment de sa retraite »

assurance retraite (1)

«Forme d'assurance qui permet de se constituer volontairement un complément de retraite »

assurance retraite (2)

« Produit d'épargne retraite »

assurance retraite (3)

« Contrat d'assurance retraite » ubezpieczenie emerytalne (1)

„Ubezpieczenie społeczne mające na celu zabezpieczenie osób w wieku emerytalnym, czyli w takim, który powszechnie uznaje się za wiek niezdolności zawodowej”

ubezpieczenie emerytalne (2)

„Ubezpieczenie indywidualne, którego głównym zadaniem jest zapewnienie osobie ubezpieczonej wypłaty świadczenia w postaci renty po osiągnięciu wieku emerytalnego"

ubezpieczenie emerytalne (3)

„Produkt emerytalny”

ubezpieczenie emerytalne (4)

„Umowa indywidualnego ubezpieczenia emerytalnego"

3.7. Il est évident que l'analyse des notions doit se faire par comparaison des éléments descriptifs, et non par similarités formelles des dénominations. Néanmoins, le rapprochement graphique et/ou phonétique propre à certains internationalismes est quelquefois un indice qui facilite la mise en relation :

$\begin{aligned} \text { accumulation } & \Leftrightarrow \text { akumulacja } \\ \text { action } & \Leftrightarrow \text { akcja } \\ \text { adhésion } & \Leftrightarrow \text { adhezja } \\ \text { allocation } & \Leftrightarrow \text { alokacja } \\ \text { clause } & \Leftrightarrow \text { klauzula }\end{aligned}$

$\begin{aligned} \text { cessionnaire } & \Leftrightarrow \text { cesjonariusz } \\ \text { dividende } & \Leftrightarrow \text { dywidenda } \\ \text { capitalisation } & \Leftrightarrow \text { kapitalizacja } \\ \text { coassurance } & \Leftrightarrow \text { koasekuracja } \\ \text { indexation } & \Leftrightarrow \text { indeksacja }\end{aligned}$

Il arrive aussi que la transposition plus ou moins linéaire des éléments constituant le terme d'une langue à l'autre puisse amener à saisir son équivalent potentiel : 


$\begin{aligned} \text { assurance dotale } & \Leftrightarrow \text { ubezpieczenie posagowe } \\ \text { ontant de prime } & \Leftrightarrow \text { wysokość składki } \\ \text { annulation du contrat } & \Leftrightarrow \text { unieważnienie umowy } \\ \text { pilier à la retraite } & \Leftrightarrow \text { filar emerytalny } \\ \text { plan retraite } & \Leftrightarrow \text { plan emerytalny } \\ \text { instrument financier } & \Leftrightarrow \text { instrument finansowy } \\ \text { apital initial } & \Leftrightarrow \text { kapitat poczatkowy } \\ \text { prestation d'assurance } & \Leftrightarrow \text { świadczenie ubezpieczeniowe } \\ \text { prime d'assurance } & \Leftrightarrow \text { sktadka ubezpieczeniowa } \\ \text { prorogation du contrat } & \Leftrightarrow \text { przedtużenie umowy }\end{aligned}$

Bien entendu, ces spécificités des terminologies, quoique alléchantes par leur simplicité d'application, ne doivent aucunement être les seules indicatrices des équivalences, car l'identité de l'orthographe n'est pas toujours compatible avec l'identité du référent, et la transposition des unités d'une langue vers l'autre n'est pas non plus garante d'une équivalence légitimement fixée. Le risque de confusion engendré par l'existence des faux-amis ou des "mots perfides", comme les dénomme Albert Barrera-Vidal (1995 : 16), existe. Par ailleurs, nous y étions également confrontée: le terme français bonus ${ }^{12}$, se définissant en assurance vie comme "participation aux bénéfices », n'a pas comme équivalent le terme polonais bonus, réservé à l'assurance automobile.

3.8. Il arrive qu'en dépit d'informations explicitement formulées qui auraient pu se retrouver circonscrites dans les fiches, ou simplement à défaut d'informations, certains termes ont été quand même juxtaposés comme équivalents ${ }^{13}$. Il est évident que ce type d'opération court le risque de ne pas saisir pleinement ou correctement le sens terminologique des unités ainsi mises en relation, et à plus forte raison de ne pas devenir une stratégie régulière. Néanmoins, puisqu'il s'agit de termes qui se faufilent dans les textes du domaine avec une certaine fréquence, les indices sur leur sémantisme s'y trouvent également. De plus, notre initiation au domaine, qui n'est plus celle d'un profane, nous a enhardie à prendre quelques décisions plus risquées; nous avons en même temps conscience que pour l'attestation de leur statut d'équivalence, ces tandems réclament sans aucun doute l'intervention d'un spécialiste ou une recherche documentaire supplémentaire. Il s'agit entre autres des termes :

${ }^{12}$ Le terme fonctionne parallèlement en assurance automobile et en assurance vie avec des sens différents et apparemment pour cette raison, en assurance vie, il y est déconseillé par la commission sur l'amélioration du langage de l'assurance.

${ }^{13}$ Il s'agit entre autres de termes qui se sont déjà infiltrés dans la langue commune et qui paraissent sémantiquement plus ou moins transparents pour tous les usagers d'une langue. 


\begin{tabular}{|c|c|c|c|c|c|}
\hline $\begin{array}{l}\text { doublement du } \\
\text { capital }\end{array}$ & $\Leftrightarrow$ & $\begin{array}{l}\text { podwójna suma } \\
\text { ubezpieczenia }\end{array}$ & $\begin{array}{l}\text { taux de rendement } \\
\text { garanti }\end{array}$ & $\Leftrightarrow$ & $\begin{array}{l}\text { gwarantowana stopa } \\
\text { zysku }\end{array}$ \\
\hline $\begin{array}{l}\text { premier bénéficiai- } \\
\text { re désigné }\end{array}$ & $\Leftrightarrow$ & główny uposażony & $\begin{array}{l}\text { assurance prévoy- } \\
\text { ance }\end{array}$ & $\Leftrightarrow$ & $\begin{array}{l}\text { ubezpieczenie o charak- } \\
\text { terze ochronnym }\end{array}$ \\
\hline prévoyance décès & $\Leftrightarrow$ & $\begin{array}{l}\text { zabezpieczenie na } \\
\text { wypadek śmierci }\end{array}$ & bancassureur & $\Leftrightarrow$ & $\begin{array}{l}\text { grupa bankowo- } \\
\text { ubezpieczeniowa }\end{array}$ \\
\hline échéance de prime & $\Leftrightarrow$ & $\begin{array}{l}\text { data wymagalności } \\
\text { sktadki }\end{array}$ & $\begin{array}{l}\text { provisions tech- } \\
\text { niques }\end{array}$ & $\Leftrightarrow$ & rezerwa techniczna \\
\hline
\end{tabular}

3.9. Par ailleurs, un autre problème paraît encore altérer la mise en équivalence et la justesse du choix opéré quant à l'indication correcte du terme dominant dans une série de termes convoqués pour désigner le même concept. Prenons l'exemple du terme français arbitrage que nous avons juxtaposé au terme polonais konwersja. Ce dernier a été sélectionné - la question est de savoir si légitimement - parmi plusieurs d'autres (tels que: przeniesienie aktywów pomiędzy funduszami, transfer środków pomiędzy funduszami, zmiana funduszu, zmiana jednostek funduszy, zmiana ubezpieczeniowych funduszy inwestycyjnych, zmiana udziałów $w$ funduszu, zmiana udziału funduszy $w$ alokowanej sktadce, przeniesienie jednostek uczestnictwa, przeniesienie środków pomiędzy funduszami), ayant la valeur de référer au concept défini ci-dessous :

arbitrage

«Opération, réalisée par le souscripteur, qui consiste à transférer tout ou partie de l'épargne gérée d'un support financier vers un autre support» konwersja

„Operacja, podczas której dokonuje się przeniesienia środków pomiędzy funduszami zarządzanymi przez to samo towarzystwo"

3.10. Finalement, il est fréquent que les termes figurant dans le fichier ne soient pas équipés de leurs équivalents. Cela induit que l'appariement d'après les données disponibles s'est avéré problématique ou impossible, soit à cause de l'insuffisance ou de la disparité des données recueillies, soit parce qu'il peut s'agir d'un vide notionnel à l'intérieur de l'un des systèmes. Une situation toute naturelle, comme le remarque Marc Van Campenhoudt (2000 : 128), quand le travail terminologique tend à confronter deux univers notionnellement autonomes, propres à deux langues différentes. Par ailleurs, depuis la sémantique structurale, on sait que les langues ne disposent pas du même nombre de concepts $^{14}$, parce que les cultures qui leurs sont propres ne possèdent pas le

\footnotetext{
${ }^{14}$ À cet endroit, il serait plus juste de parler des signifiés.
} 
même niveau de connaissances. De la sorte - une donnée relevant déjà du relativisme linguistique - le développement d'une langue est inhérent au développement de la culture et de la civilisation où cette langue opère; dans le cas des langues de spécialité, au développement de la discipline qui leur est exclusive.

Les disparités systémiques identifiées concernent avant tout les concepts désignant les réalités propres à des initiatives législatives ou économiques, inhérentes à chaque pays. Par conséquent, la présence dans un système d'un tel concept asymétrique provoque l'apparition d'un vide notionnel dans l'autre. Il est toutefois intéressant que parmi les termes désignant des concepts « internes », les termes français sont infiniment nombreux ; cela confirmerait une plus grande «maturité » du système français, ainé par rapport au système polonais. Car, précisons-le, le système par piliers a été instauré en France peu après la seconde guerre mondiale, en subissant depuis de nombreux ajustements correctifs. La mise en place du système polonais, inspiré de modèles occidentaux, ne remonte officiellement qu'à 1999. Certes, il ne faut pas prendre cette date au pied de la lettre, d'autant plus que la terminologie du troisième pilier exploite largement les fonds terminologiques des disciplines connexes, telles que l'assurance ou l'économie. Néanmoins, ce décalage temporel ne peut pas rester sans impact sur la matière terminologique confrontée. En conséquence, les termes français non appariés désignent notamment :

a) les produits d'assurance-retraite : ACAVI, assurance combinée, assurance de capital différé, assurance vie entière différée, Caps, contrat dit "Article 39 », contrat DSK, contrat Madelin, contrat NSK, contrat PEP, FCP, PEP bancaire, PEP assurance, etc. ;

b) les organismes gestionnaires : AGIRC, ARRCO, caisse du régime, caisse professionnelle, fonds de fonds, etc.;

c) les pratiques, procédures ou opérations agrégées : acceptation du bénéficiaire, acte d'assureur, cantonnement, contre-assurance, effet de cliquet, prélèvement libératoire, titre de retraite, plus-value latente, précompte de commission, préliquidation, prime viagère, prorata temporis, etc.

\section{CONCLUSION}

La dimension bilingue d'un corpus pose inévitablement plusieurs problèmes. Ces quelques situations décrites de manière non exhaustive renseignent sur les similarités et les disproportions existant entre les deux systèmes notionnels mis en confrontation. Il va de soi qu'au niveau d'équivalence ces dernières posent beaucoup plus de problèmes; mais par là, elles font rappeler une évidence 
flagrante : admettre qu'une notion soit commune à tous les locuteurs ou unanimement par eux saisie, quelle que soit leur langue, relèverait d'un raisonnement simpliste. Les divergences entre les systèmes notionnels sont tant naturels qu'inéluctables; pourtant, leur caractère et leur volume sont des données variables et changent au fil du temps d'une langue à l'autre, d'une discipline à l'autre. Dans les disciplines abstraites, telle que la nôtre, cet état de fait est particulièrement remarquable. Et puisque la mise en équivalence se fait par comparaison des systèmes, la pénurie de similitude est souvent interprétée comme symptôme d'imperfection, voire d'immaturité d'un système en une langue par rapport à son homologue dans l'autre. Bien que cette donnée soit particulièrement juste dans le cas des terminologies étudiées, elle n'est certainement pas la seule source des inégalités systémiques. Les carences et les disparités entre les systèmes sont une donnée universelle et objective. Peut-on donc dans ces conditions aboutir au stade de l'équivalence pleinement satisfaisante ? Une question qui malgré l'incontestable unité du domaine en deux langues, certes régie par des lois différentes, relèverait paradoxalement d'un idéalisme incorrect ; de l'idéalisme tant reproché de nos jours à Wüster. 


\section{BIBLIOGRAPHIE}

Barrera-Vidal, A. (1995). La paronymie dans l'apprentissage de l'espagnol par des francophones : applications à la compétence de lecture en ELE. In J. Sypnicki (Éds.), Polysémie, synonymie, antonymie. Relations dans le lexique. Aspects théoriques et applicatifs (pp. 5-24). Łódź : UŁ.

Bejoint, H. \& Thoiron, Ph. (2000). Le sens des termes. In H. Béjoint \& Ph. Thoiron (Éds.), Le sens en terminologie (pp. 5-19). Lyon : Presse Universitaire de Lyon.

Depecker, L. (2000). Le signe entre signifié et concept. In H. Béjoint \& Ph. Thoiron (Éds.), Le sens en terminologie (pp. 86-126). Lyon : Presse Universitaire de Lyon.

Gouadec, D. (1990). Terminologie. Constitution des données. Paris : Afnor.

Gouadec, D. (1993). Terminologie \& terminotique. Outils, modèles \& méthodes. Paris : La maison du dictionnaire.

Kaufman, S. (2002). Problemy ekwiwalencji terminologicznej. In R. Lewicki (Éds.), Przekład, język, kultura (pp. 161-169). Lublin: Wydawnictwo UMCS.

Kaufman, S. (2004). Le microsystème notionnel du tourisme et sa description terminologique. Romanica Wratislaviensia, LI, 667, 49-73.

Kozłowska, Z. (2007). O przekładzie tekstu naukowego (na materiale tekstów językoznawczych). Warszawa: UW.

Lukszyn, J. \& Zmarzer, W. (2006). Teoretyczne podstawy terminologii. Warszawa: UW.

Rey, A. (1992). Terminologie. Noms et notions. Paris : PUF.

Van Campenhoudt, M. (1995). Le réseau notionnel interlinguistique. Réseau notionnel, intelligence artificielle et équivalence en terminologie multilingue: essai de modélisation. Communication aux IV journées scientifiques du réseau L.T.T. Lyon. www.termisti.refer. org/rni.htm

Van Campenhoudt, M. (2000). De la lexicographie spécialisée à la terminographie : vers un « métadictionnaire »? In H. Béjoint \& Ph. Thoiron (Éds.), Le sens en terminologie (pp. $127-$ 152). Lyon : Presse Universitaire de Lyon. 